\title{
ANALYSIS OF VORTEX STRUCTURE IN A SHALLOW STRATIFIED LAKE
}

\author{
Junior Researcher Olga S. Volodko ${ }^{1,2}$ \\ Senior Researcher Lidiya A. Kompaniets ${ }^{1,2}$ \\ Assist. Prof. Lyudmila V. Gavrilova ${ }^{2}$ \\ ${ }^{1}$ ICM SB RAS, Krasnoyarsk, Russia \\ ${ }^{2}$ Siberian Federal University, Krasnoyarsk, Russia
}

\begin{abstract}
In the summer period of 2018 satellite-tracked quasi-lagrangian drifter was launched in saline stratified Lake Shira (Republic of Khakassia, Russia). The analysis of obtained data was revealed a mesoscale vortex structure in the lake.

At the same time, measurements of the current velocity were carried out at several points in the lake using an Acoustic Doppler Current Profiler (ADCP).

The reliability of the data obtained using the quasi-lagrangian drifter is confirmed by a comparison with the data for the current velocity and for the direction and speed of the wind.
\end{abstract}

The horizontal velocity was analyzed using complex empirical orthogonal functions. The hodograph of the first temporal coefficient shows the elliptical structure of the current. This effect may be due to the strong variability of wind on the lake during the summer period or to the vortex structure of wind stress.

To clarify the origin and distribution of the mesoscale eddies (vortices) in Lake Shira, it is necessary to conduct a study with a large number of drifters.

Keywords: lagrangian drifters, ADCP, mesoscale eddies, lake circulation

\section{INTRODUCTION}

The vortex structures carry out an important role in the process of forming currents in open bodies of water. Since vortices are source of energy transfer of internal waves, they significantly contribute to the horizontal heterogeneity of phyto- and zooplankton distribution.

In particular, the problems of study of these phenomena were discussed in the International Symposium on Mesoscale and Submesoscale Processes in the Hydrosphere and the Atmosphere (MSP - 2018).

Numerous investigations focused on the study vortex processes in the atmosphere and ocean. The study of these effects in lakes is conducted much less. For example, the study of mesoscale vortex structure in the Lake Stechlin was carried out in the paper [1]. Using four quasi-lagrangian drifters, the authors revealed a moving vortex in the lake in November 2004. 
In this paper, the possibility of the appearance of vortex structures in the Lake Shira is studied by using quasi-lagrangian drifter, the instruments for measurements of current velocity and analysis of wind direction. This lake is located in the Republic of Khakassia and is the subject of investigation in view of the great importance of this subject for this region.

\section{MATERIALS AND METHODS}

In the summer period of 2018 satellite-tracked quasi-lagrangian drifter was launched in the saline stratifiedd Lake Shira (Republic of Khakassia, Russia) by the researchers from the Institute of Biophysics of Siberian Branch of Russian Academy of Sciences. At the same time, measurements of the current velocity were carried out at several points in the lake using an ADCP (Fig. 1).

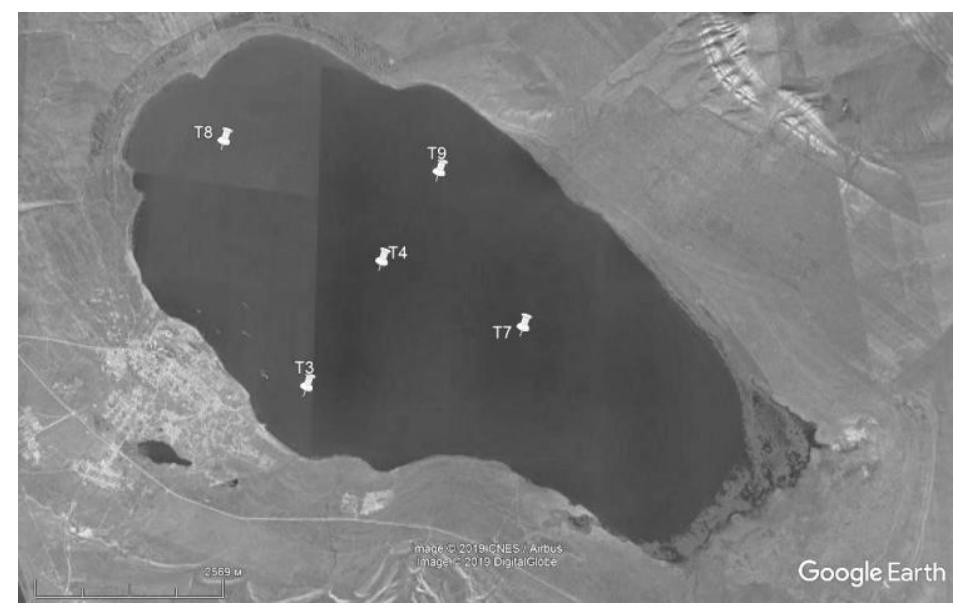

Figure 1. ADCP locations in summer 2018.

The tracked quasi-lagrangian drifter was deployed from the location T4.

\section{RESULTS}

Fig. 2 shows by a continuous line the trajectory of the movement of drifter during the experiment, dots on the line indicate the time when the wind changed the direction. The beginning of the experiment was in 12/07/18 at 7:22. The duration of the experiment was 23 hours. The average speed of the drifter is $9 \mathrm{~cm} / \mathrm{s}$.

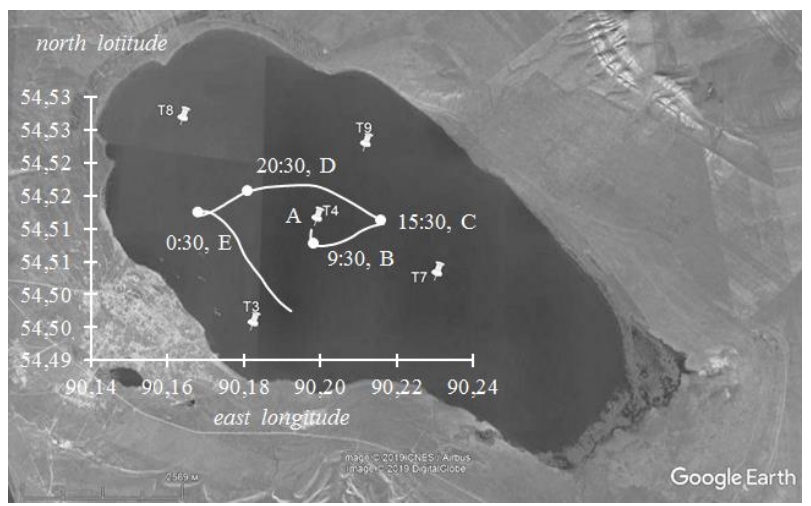

Figure 2. The trajectory of the movement of drifter in June 12-13, 2018. 
To analyze the drifter movement the data of wind direction and speed (Fig. 3) from a meteorological station located on the shore of Lake Shira about $2 \mathrm{~km}$ from the start of the drifter were used.
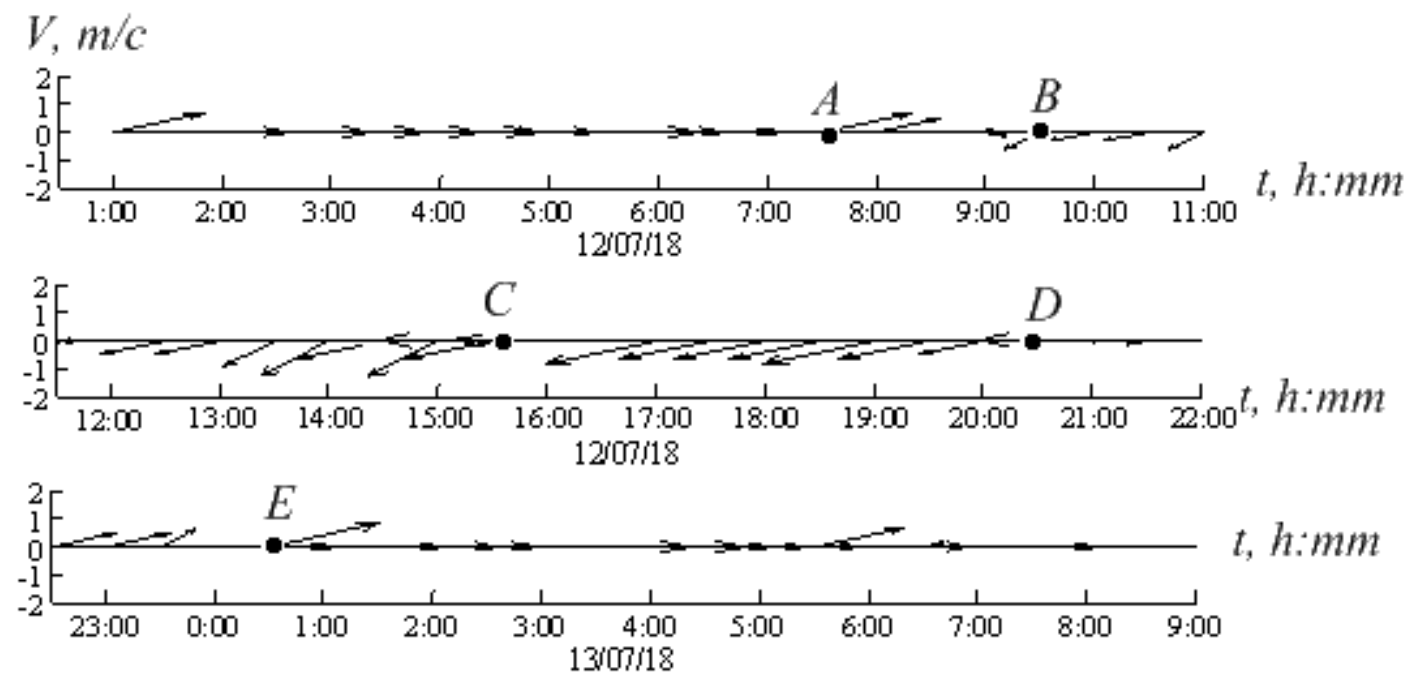

Figure 3. Wind direction and speed in 12/07/18 -13/07/18

According to Fig. 3 it can be seen that before the start of the experiment the wind had a westerly direction, after the start of the experiment (Fig. 3, point A) the wind had westsouth-west direction a short time, then it changed direction to east-north-east. After 20:30 07/12/18 (fig. 3, point D) the wind again changed direction and until the end of the experiment it blew mainly from the west. Comparing Figure 3 with Figure 2, one can see that the change in wind direction leads to the change of drifter trajectory.

The Fig. 4 shows the results of measuring of the current velocity by ADCP instrument in the location of the start of the drifter.

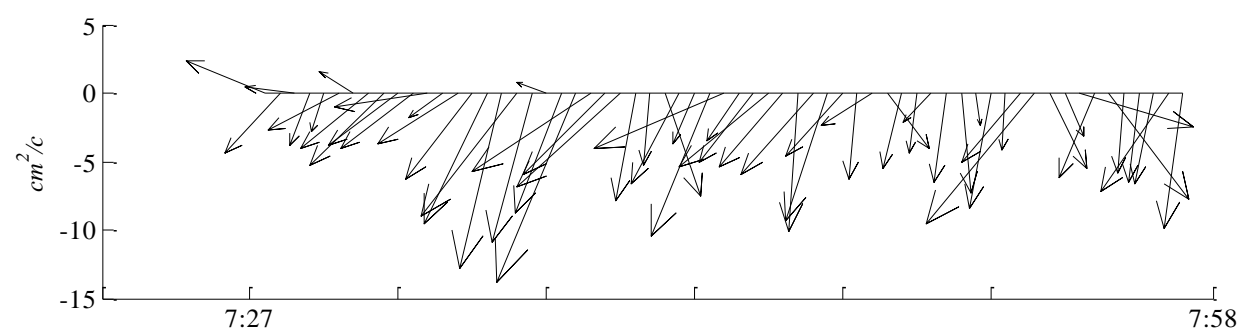

Figure 4. The direction and velocity of flow on the surface of lake at the point T4 measured by the ADCP in 12/07/18

The Fig. 5 shows the direction and magnitude of the surface velocity measured by $\mathrm{ADCP}$ at other locations. 

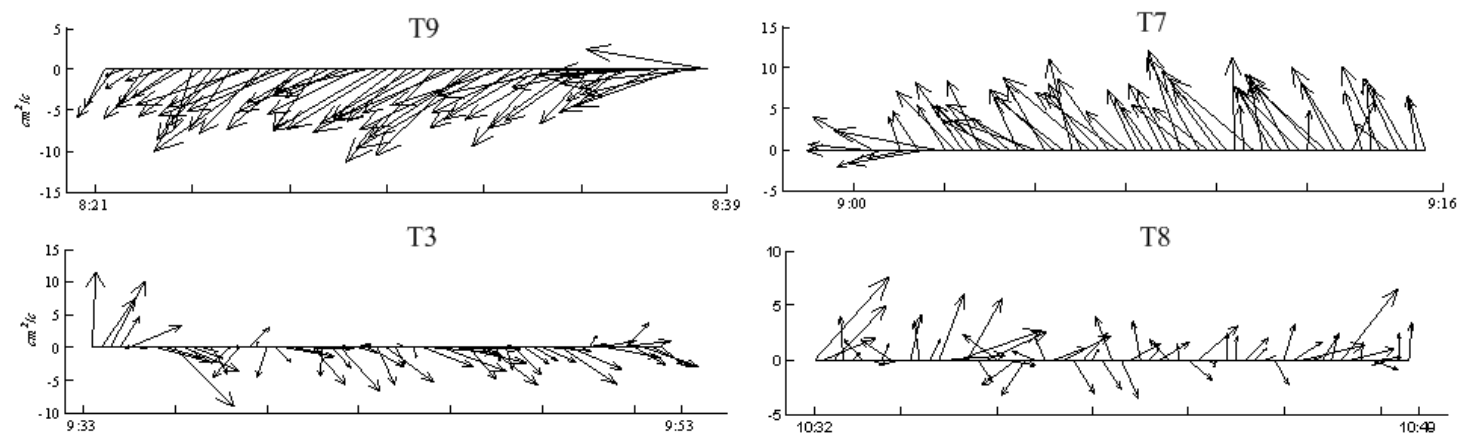

Figure 5. The direction and values of the surface velocity at locations T3, T7, T8, T9 measured by the ADCP 12/07/18

The measurements at T3, T4, T7, T8, T9 locations were conducted at different moments during 2,5 hours, but we considered them as if measures were made at the same time. This allows to use the method of empirical orthogonal functions to determine the main components of horizontal velocity [2-3]. The Figure 6 shows the part of accumulated energy. The Figure 7 shows the first mode and corresponding modal coefficient.

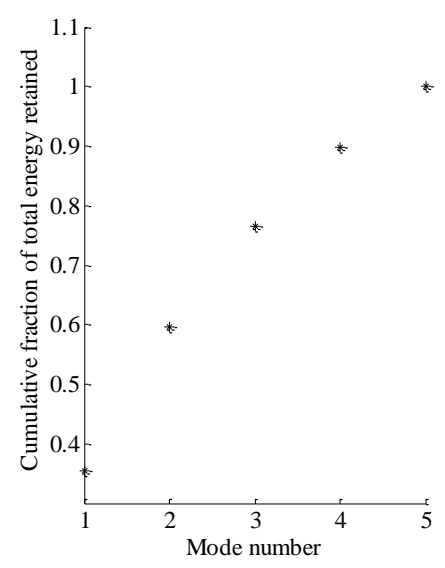

Figure 6. The accumulated part of energy.
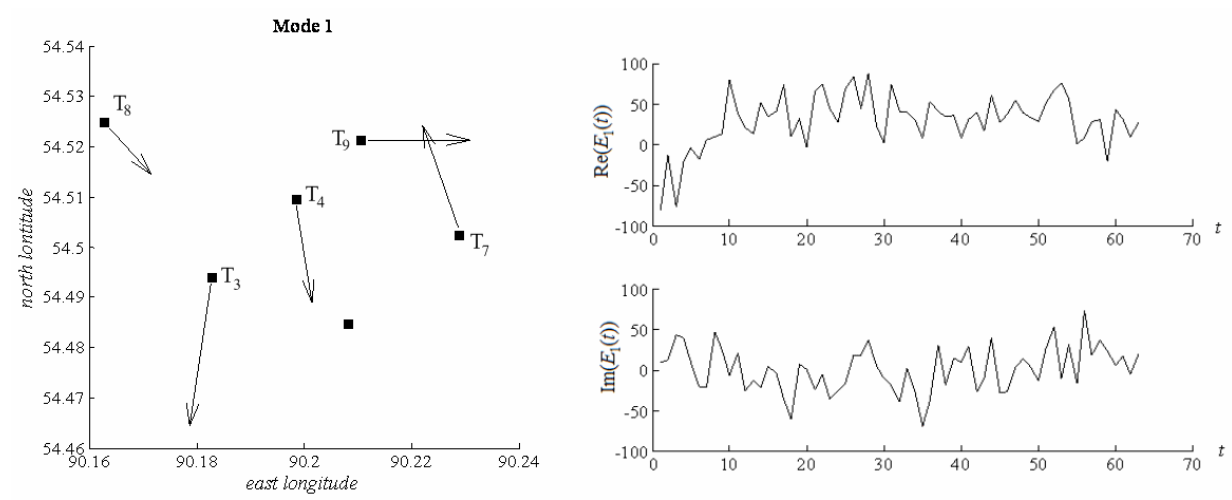

Figure 7. The first mode and the first modal coefficient of horizontal velocity.

This velocity distribution does not contradict to the motion of drifter at the initial moment of time (after the start at 7.22 to 9.30 ). 
The possibility of the vortex structure of flow specified by drifter can be explained also by wind picture at the area of the Lake Shira. The data of direction and speed of wind from several meteorological stations located around the Lake Shira were considered (Fig. 8).

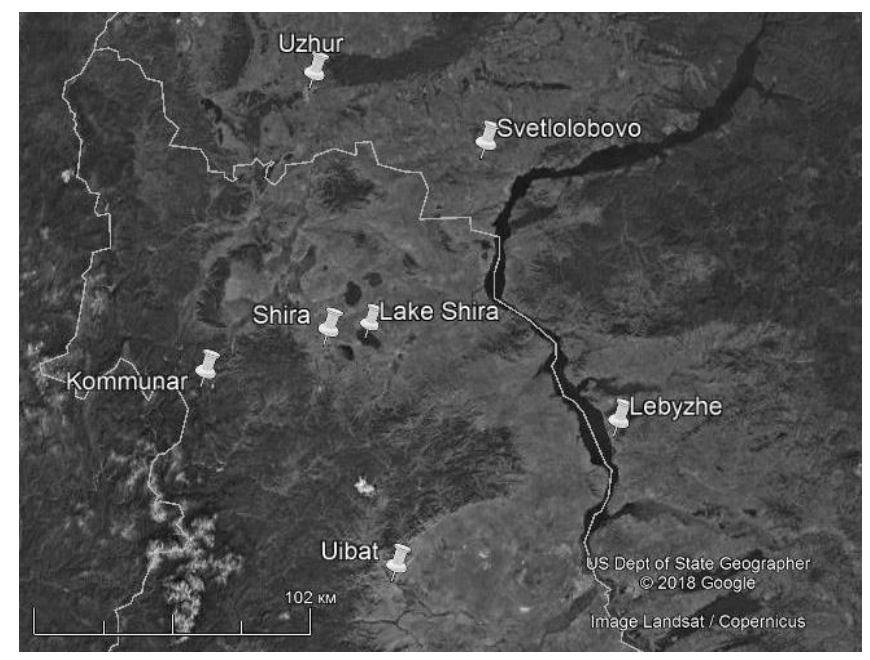

Figure 8. Meteorological stations are located near Lake Shira.

The data from meteorological stations located at the vertices of triangle circumscribed about the lake were considered for analysis.

The following triangles were considered: Uzhur - Lebyazhye - Shira, Svetlolobovo Uibat - Shira, Svetlolobovo - Lebyazhye - Shira, Svetlolobovo - Uibat - Kommunar.

Further, a linear interpolation of the projection of the wind in the direction of the axes $\mathrm{Ox}$ and $\mathrm{Oy}$ of the Cartesian coordinate system was performed according to the values at the vertices of the triangle. The wind speed rotor was found using obtained wind interpolation.
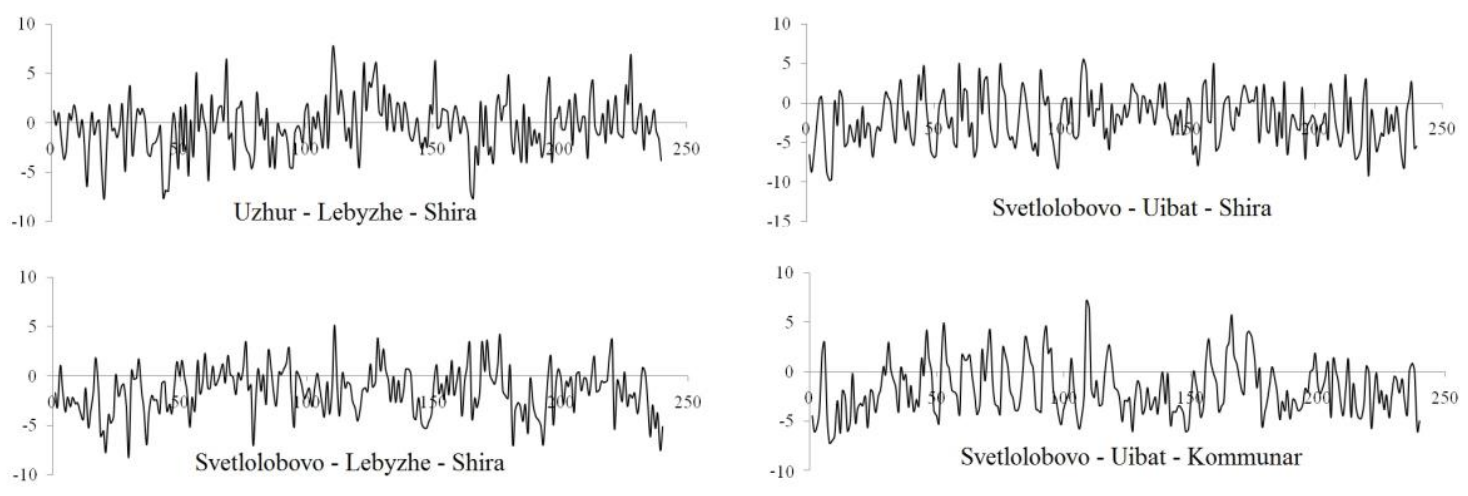

Figure 9. Wind vorticity change with time over the measurement period from 01/07/18 to $31 / 07 / 18$. 

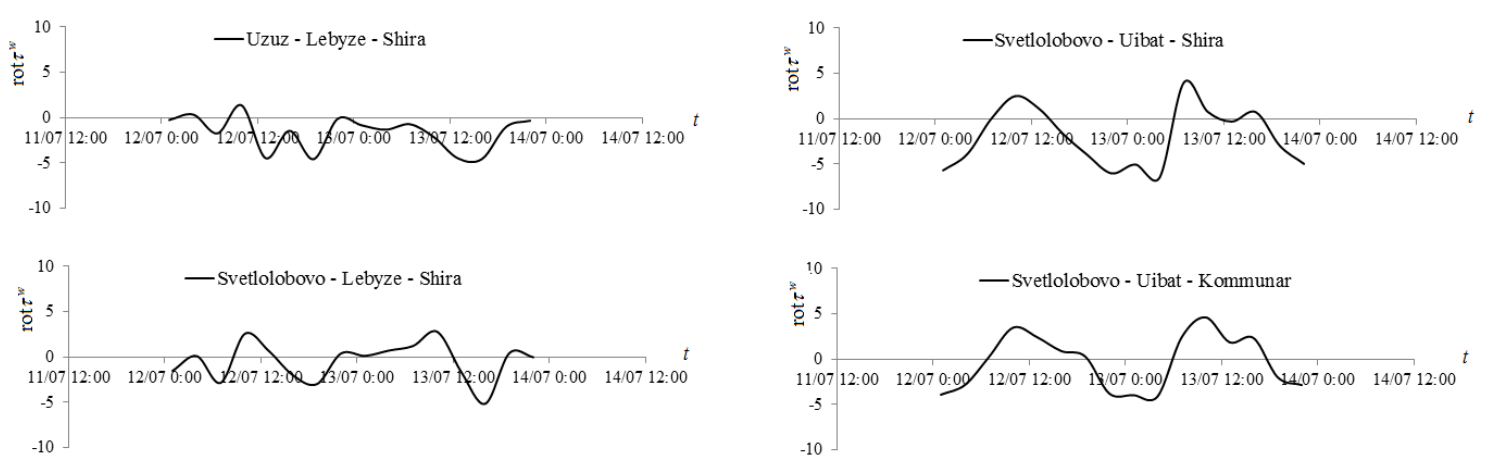

Figure 10. Wind vorticity evolution during the period of experiment with drifter from $12 / 07 / 18$ to $13 / 07 / 18$

The correlation between the values of wind vorticity was high in two cases. The first case was when the Uzhur-Lebyazhie-Shira and Svetlolobovo - Lebyazhye - Shira stations were considered during the measurement period from 01/07/18 to 31/07/18 and during the measurement period from 12/07/18 to 13/07/18. The second case was when Svetlolobovo - Uibat - Shira and Svetlolobovo - Uibat - Kommunar stations were considered during the measurement period from $01 / 07 / 18$ to $31 / 07 / 18$ and during the measurement period from 12/07/18 to 13/07/18.

The importance of study of wind exposure is related to the fact that a vortex flow is produced if the value of vorticity of the velocity vector is non-zero. This fact is shown by analytical solutions of simplified geophysical equations.

Let's consider the system of Ekman equations [4] of a steady flow of a homogeneous fluid in a Cartesian coordinate system $O x y$

$$
\begin{gathered}
-f v=-g \frac{\partial \zeta}{\partial x}+K_{z} \frac{\partial^{2} u}{\partial z^{2}} \\
f u=-g \frac{\partial \zeta}{\partial y}+K_{z} \frac{\partial^{2} v}{\partial z^{2}} \\
\frac{\partial u}{\partial x}+\frac{\partial v}{\partial y}+\frac{\partial w}{\partial z}=0 .
\end{gathered}
$$

Here $u, v, w$ are horizontal and vertical current velocities, $f$ is Coriolis parameter, $K_{z}$ is coefficient of vertical turbulent exchange, $g$ is acceleration of gravity, $\zeta$ is the free surface elevation. On the lateral boundary the normal component of the total flow is zero. On the bottom there is no-slip condition. On the unperturbed free surface, $\tau^{x}, \tau^{y}$ are surface wind stress along the axes $O x, O y$ respectively.

The solution for the drift component was written in [5]. The slopes of the free surface are found using the current function from the equation

$$
\frac{\partial^{2} \Phi}{\partial x^{2}}+\frac{\partial^{2} \Phi}{\partial y^{2}}=\frac{1}{f}\left(\left(C+D \frac{F}{E}\right) \operatorname{curl}\left(\tau^{w}\right)+\left(D-C \frac{F}{E}\right) \operatorname{div}\left(\tau^{w}\right)\right),
$$




$$
\operatorname{curl}\left(\tau^{w}\right)=\frac{\partial \tau_{y}^{w}}{\partial x}-\frac{\partial \tau_{x}^{w}}{\partial y}, \quad \operatorname{div}\left(\tau^{w}\right)=\frac{\partial \tau_{x}^{w}}{\partial x}+\frac{\partial \tau_{y}^{w}}{\partial y} .
$$

The coefficients $C, D, E, F$ are written in [5].

If the wind stress is given by the formula $\tau_{x}^{w}=-y, \quad \tau_{y}^{w}=x$, then in the case of a circular cylinder of radius $R$ the function $\Phi$ is written out in the final form, in the case of a rectangular basin this function is found in the form of a Fourier series.

The Fig. 10 shows the case of velocity field form under following parameters:

$R=625 \mathrm{~m}$ and depth of $50 \mathrm{~m}$, the Coriolis parameter $f=0,00015 \mathrm{~s}^{-1}, K_{z}=0,02 \mathrm{~m}^{2} / \mathrm{s}$.
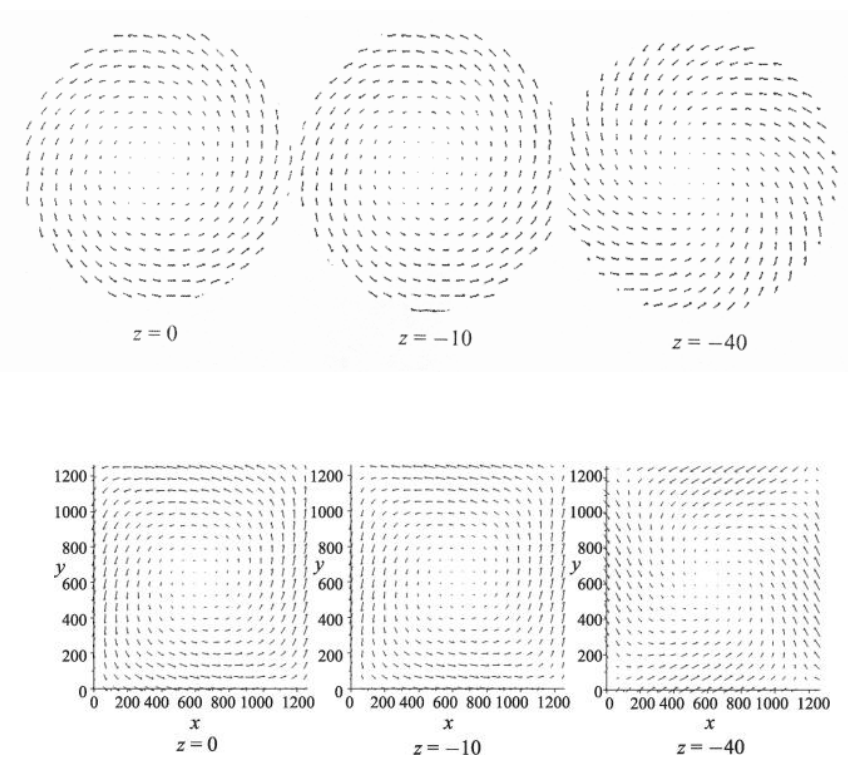

Figure 10. On the top is velocity field in the basin of cylinder form, on the bottom is velocity field in the basin of rectangular form.

In both cases, such wind exposure leads to the formation of vortex structures.

\section{CONCLUSION}

The observations of recent years have revealed significant velocity variability in the Lake Shira in the summer. The instruments of the ADCP series, which allows long-term measurements at one location at short intervals were used for the analysis of velocity. It was found the horizontal velocity performed a full turn at the measuring location. The interpretation of this fact was difficult due to the imperfection of the theoretical description and the difficulty of experimental observations.

The lagrangian drifter was used for the first time in the summer measurement of 2018, what made it possible to reveal the presence of vortex formations in the lake. The question of the nature of their formation remains open: it can be the destruction of the internal wave or the influence of vortex structure of wind. 
Analysis of the wind pattern for the three weather stations shows that the second case is also possible. In this case, wind stress is a linear function of spatial variables with nonzero vorticity, and as the analytical solution for the simplified Ekman model of threedimensional fluid flow shows, this leads to the formation of a vortex.

To clarify the trajectory of the vortex, it is planned to conduct in-situ observations with several drifters in 2019.

\section{REFERENCES}

[1] Kirillin G., Golosov S. A mesoscale vortex in a small stratified lake //Environ Fluid Mech. 8. pp. 349-366, 2008.

[2] Lorenz E.N., Empirical orthogonal functions and statistical weather prediction //Sci. Rep. No. 1, Statistical Forecasting Project, M.I.T., Cambridge, MA, 1956, p 48.

[3] Kundu P.K., Allen J.S., Smith R.L., Modal decomposition of the velocity field near the Oregon Coast //Journal of Physical Oceanography, USA, vol. 5/issue 4, pp. 683704, 1975.

[4] Ekman, V. W. On the influence of the Earth rotation on ocean currents //Arkiv Mat., Astron., Fysik. - 1905. - Bd. 2. - № 11. - S. 1-52

[5] Welander P. Wind Action on a Shallow Sea: Some Generalizations of Ekman's Theory // Institute of Theoretical Physics, University of Stockholm, and International Meteorological Institute in Stockholm, 1956. - C.45-52. 